\title{
RIS-aided Hybrid Massive MIMO Systems Relying on Adaptive-Resolution ADCs: Robust Beamforming Design and Resource Allocation
}

\author{
Yalin Wang, Xihan Chen, Yunlong Cai, Senior Member, IEEE, and Lajos Hanzo, Fellow, IEEE
}

\begin{abstract}
The large-scale multiple-input multiple-output (MIMO) uplink is investigated in the presence of channel-induced uncertainty, where variable-resolution analog-to-digital converters (ADCs) are used at the base station (BS) and a reconfigurable intelligent surface (RIS) is employed for supporting communications between the single-antenna users and the multiantenna BS. We formally maximize the system throughput by jointly optimizing the ADC's resolution, the transmit power, the passive reflection coefficients of the RIS and the hybrid combiner of the BS subject to practical constraints under statistical cascaded channel state information (CSI) error model. The robust nonconvex optimization problem is firstly decoupled via the classic Lagrangian dual transform and fractional programming method, followed by a powerful decoupling-based alternating maximization (D-AltMax) algorithm to solve this challenging problem. Our simulation results reveal the supremacy of our proposed algorithm over the benchmark schemes by quantifying the improved system throughput of this robust design.
\end{abstract}

Index Terms-Massive multiple-input multiple-output (MIMO), reconfigurable intelligent surface (RIS), fractional programming, alternating optimization, robust beamforming design, imperfect CSI.

\section{INTRODUCTION}

As a promising candidate for the next generation of wireless communication networks, massive multiple-input multipleoutput (MIMO) schemes are capable of significantly enhancing the spectral/energy efficiency attained [1]. However, massive MIMO systems adopting traditional fully-digital processing using power-hungry high-resolution analog-to-digital converters (ADCs) increase the complexity as well as the cost of their practical implementation. Hence the concept of hybrid analog-digital beamforming architectures relying on low-resolution ADCs (LADCs) was conceived, which reduces the number of radio frequency (RF) chains and hence the power dissipation. To unleash the full potential of such architectures, there has been a number of studies on beamforming optimization [2] and user scheduling [3], which significantly increase the system's rate and/or energy efficiency [1], [4]. Unfortunately, under unfavorable propagation conditions, especially in millimeter wave (mmWave) systems operating at high-frequencies the link between the transmitter and receiver

Copyright (c) 2015 IEEE. Personal use of this material is permitted. However, permission to use this material for any other purposes must be obtained from the IEEE by sending a request to pubs-permissions@ieee.org.

The work of Y. Cai was supported in part by the National Key R\&D Program of China (No.2020YFB1805005), the National Natural Science Foundation of China under Grants 61971376 and 61831004, and the Zhejiang Provincial Natural Science Foundation for Distinguished Young Scholars under Grant LR19F010002. L. Hanzo would like to acknowledge the financial support of the Engineering and Physical Sciences Research Council projects EP/P034284/1 and EP/P003990/1 (COALESCE) as well as of the European Research Council's Advanced Fellow Grant QuantCom (Grant No. 789028). (Corresponding author: Yunlong Cai.)

Y. Wang, X. Chen, and Y. Cai are with the College of Information Science and Electronic Engineering, Zhejiang University, Hangzhou 310027, China (e-mail: \{wang_yalin, chenxihan, ylcai\}@zju.edu.cn). L. Hanzo is with the Department of ECS, University of Southampton, Southampton SO17 1BJ, U.K. (e-mail: 1h@ecs.soton.ac.uk). might be susceptible to blockages, which makes their communications inefficient.

To mitigate the effect of blockages, the cost- and powerefficient reconfigurable intelligent surface (RIS) concept has been proposed for remarkably improving the wireless communication performance [5]. Specifically, RIS is a reflecting/refracting surface composed of numerous low-cost passive elements, each of which is capable of adjusting the amplitude and/or phase of the incident signal independently via a controller connected to the base station (BS). Owing to the above benefits, substantial research efforts have been invested in the design of RIS-aided communication systems, including full-duplex systems [6], unmanned aerial vehicle (UAV) aided [7], [8] and mmWave communication [9]-[14], etc. In [9], the authors exploited the refraction capability of RISs and developed a RIS-tiled wall for intelligently transitting the signal from an outdoor BS to indoor user equipment, which increases the chance of high-integrity outdoor-to-indoor communication. As a further advance, in [12], the authors harnessed the RIS as a reflection-type surface and studied a point-to-point RIS-aided mmWave system relying on hybrid precoding/combining, where a manifold optimization based algorithm was developed for maximizing the spectral efficiency. In [14], the authors focused their attention on the hybrid precoding design of a RIS-aided multiuser mmWave system and formulated a mean-squared-error (MSE) minimization problem, which was solved by employing the gradient projection method. However, all these contributions rely on high-resolution quantization, which incur potentially excessive power dissipation.

There is however a paucity of contributions on RIS-aided systems adopting LADCs [15]-[17]. In [15], the authors studied the capacity of beamforming using LADCs and RIS. In [16] the authors focused their attention on the attainable rate of the RIS-aided mmWave uplink, where both LADCs and RIS phase noise were considered. As a further development, the authors of [17] investigated the uplink of RIS-assisted mmWave systems employing adaptive-resolution ADCs (RADCs). The system's achievable rate was maximized by jointly optimizing the beam selection matrix, the RIS coefficients, and the ADC resolution under realistic hardware constraints. However, the authors of [15]-[17] studied a fixed power allocation scheme, which does not exploit the potential of per-user power control in mitigating the interference. Moreover, perfect channel state information (CSI) was assumed to be available at the BS, which is impractical, since no signal processing can be used at the passive RIS. To the best of our knowledge, the robust beamforming design and power control of RIS-aided hybrid massive MIMO systems using adaptive-resolution quantization and realistic imperfect CSI have not been reported as yet.

Motivated by these observations, we conceive joint robust 


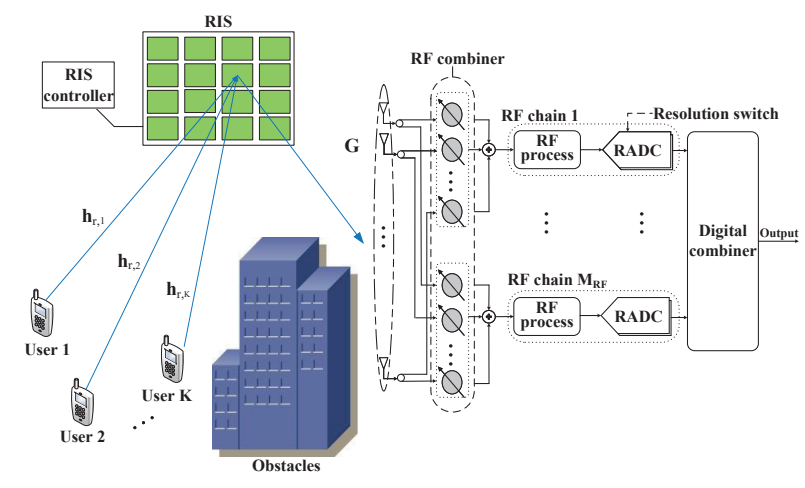

Fig. 1. An illustration of the RIS-aided uplink multiuser hybrid massive MIMO system.

beamforming and resource allocation for RIS-aided hybrid massive MIMO systems in the presence of imperfect cascaded BS-RIS-user CSI, where the RIS acts as a reflective surface, assisting in bypassing obstacles. Specifically, we aim for maximizing the worst-case sum rate via jointly optimizing the transmit power, bit allocation per ADC, hybrid combining matrix at the BS and passive beamforming matrix at the RIS. In order to solve the nonconvex optimization problem formulated, we develop a low-complexity decoupling-based alternating maximization (D-AltMax) algorithm having guaranteed convergence. Specifically, with the aid of Lagrangian dual transform and fractional programming, we equivalently transform the original problem into a mathematically tractable form, and then solve the resultant problem in an alternating manner. Our simulation results demonstrate the supremacy of the proposed scheme over the benchmark schemes.

Notations: The upper bold letters are used for matrices and the lower bold letters are used for vectors. $(\cdot)^{T}$ and $(\cdot)^{H}$ denote the transpose and conjugate transpose, respectively. $\|\cdot\|_{1}$ refers to the $\ell 1$-norm operation of vectors. $\Re\{\cdot\}$ stands for the real part of a complex number. $\operatorname{diag}(\cdot), \operatorname{Tr}\{\cdot\}$, and $\mathbb{E}[\cdot]$ denote the diagonal, trace and expectation operators, respectively.

\section{System Model and Problem Formulation}

As shown in Fig. 1, we consider the uplink of an RISaided multiuser massive MIMO system. The BS is equipped with a uniform linear array (ULA) of $M_{r}>1$ antennas and $M_{R F} \ll M_{r}$ RF chains. Each RF chain is followed by a pair of variable-resolution ADCs, which substantially reduces both the power consumption and hardware cost. We assume that $K$ single-antenna users (denoted by $\mathcal{K} \triangleq\{1, \cdots, K\}$ ) are distributed in the cell and can be served simultaneously by the BS. An RIS composed of $N=N_{R} \times N_{R}$ reflecting elements (denoted by $\mathcal{N} \triangleq\{1, \cdots, N\}$ ) arranged in the form of a uniform planar array (UPA) is employed for assisting communications between the users and the BS.

We assume that the direct links between the users and the BS are blocked by potential obstacles (e.g., high buildings) and neglected due to unfavorable propagation conditions. The channels spanning from user $k$ to the RIS and from the RIS to the BS are denoted by $\mathbf{h}_{r, k} \in \mathbb{C}^{N \times 1}$ and $\mathbf{G} \in \mathbb{C}^{M_{r} \times N}$, respectively. We also assume that all the channels experience quasistatic flat fading and remain approximately constant in each fading block. Let us define a diagonal matrix $\boldsymbol{\Phi}=\operatorname{diag}(\varphi)$ as the reflection coefficient matrix adopted at the RIS, where we have $\varphi \triangleq\left[\varphi_{1}, \cdots, \varphi_{N}\right]^{T}$ with $\varphi_{n}=c_{n} e^{j \theta_{n}}, \forall n \in \mathcal{N}$. $c_{n} \in[0,1]$ and $\theta_{n} \in[0,2 \pi)$ denote the amplitude coefficient and phase shift of the $n$-th reflection element, respectively. In this paper, the amplitude coefficient of each reflection element is set to $c_{n}=1$ for the ease of passive beamforming design. Then it follows that $\left|\varphi_{n}\right|=1, \forall n \in \mathcal{N}$.

Let $s_{k}$ represent the symbol transmitted by user $k$. Without loss of generality, $s_{k}$ is modeled by an independent and identically distributed random variable with zero mean and unit variance, i.e., $s_{k} \sim \mathcal{C N}(0,1)$. Further, we define $\mathbf{s} \triangleq\left[s_{1}, \cdots, s_{K}\right]$ being the transmit signal vector, $\mathbf{H}_{r} \triangleq$ $\left[\mathbf{h}_{r, 1}, \cdots, \mathbf{h}_{r, K}\right] \in \mathbb{C}^{N \times K}$ being the channel matrix between the RIS and users, and $\mathbf{P} \triangleq \operatorname{diag}(\mathbf{p})$ with $\mathbf{p}=\left[p_{1}, \cdots, p_{K}\right]^{T}$ being the transmit power matrix, where $p_{k}$ represents the transmit power of user $k$. Then, with the support of the reflection operation by the RIS, the signal received by the BS can be written as

$$
\mathbf{y}=\sum_{k=1}^{K} \mathbf{G} \boldsymbol{\Phi} \mathbf{h}_{r, k} \sqrt{p_{k}} s_{k}+\mathbf{z}=\mathbf{G} \boldsymbol{\Phi} \mathbf{H}_{r} \mathbf{P}^{\frac{1}{2}} \mathbf{S}+\mathbf{z},
$$

where $\mathbf{z} \in \mathbb{C}^{M_{r} \times 1} \sim \mathcal{C N}\left(0, \sigma_{0}^{2} \mathbf{I}\right)$ denotes the additive white Gaussian noise (AWGN) with corresponding variance $\sigma_{0}^{2} \mathbf{I}$.

Analog Combiner (Active analog beamforming): The received signal $\mathbf{y}$ is first combined via the analog combiner $\mathbf{W} \in \mathbb{C}^{M_{R F} \times M_{r}}$. Since phase-shifters are used to realize the analog combiner, the entries of $\mathbf{W}$ are restricted to satisfy the element-wise constant-modulus constraint, namely, $|\mathbf{W}(m, i)|=1, \forall m \in\left\{1, \cdots, M_{R F}\right\}, i \in\left\{1, \cdots, M_{r}\right\}$. Therefore, the resulting signal is given by

$$
\overline{\mathbf{y}}=\mathbf{W G} \boldsymbol{\Phi} \mathbf{H}_{r} \mathbf{P}^{\frac{1}{2}} \mathbf{s}+\mathbf{W z} .
$$

Quantization: We consider that an ADC pair is connected with an RF chain and at each RF chain the ADCs can operate with different resolutions adaptive to the propagation characteristics, which provides additional flexibility and reduced quantization error. Specifically, the in-phase and quadrature component of $\bar{y}_{m}$ (the $m$-th entry of $\overline{\mathbf{y}}$ ) is quantized at ADC $m$ with $b_{m}$ quantization bit. For analytical tractability, we adopt an additive quantization noise model (AQNM) for obtaining a linearized approximation of the quantization process. After quantizing $\overline{\mathbf{y}}$, we obtain the signal as follows

$$
\mathbf{y}_{q}=\mathcal{Q}(\overline{\mathbf{y}})=\mathbf{Q}_{a} \mathbf{W G} \mathbf{\Phi} \mathbf{H}_{r} \mathbf{P}^{\frac{1}{2}} \mathbf{S}+\mathbf{Q}_{a} \mathbf{W} \mathbf{z}+\mathbf{z}_{q},
$$

where $\mathcal{Q}(\cdot)$ stands for the quantization operator and $\mathbf{Q}_{a}$ is the diagonal matrix defined as $\mathbf{Q}_{a}=\operatorname{diag}\left(\left[1-a_{1}, \cdots, 1-\right.\right.$ $\left.\left.a_{m}, \cdots, 1-a_{M_{R F}}\right]\right)$. The normalized quantization error $a_{m}$ can be approximated as $a_{m}=\frac{\pi \sqrt{3}}{2} 2^{-2 b_{m}}$ for $b_{m}>5$. For $b_{m} \leq 5$, the value of $a_{m}$ is shown in [4, Table I]. Vector $\mathbf{z}_{q} \sim$ $\mathcal{C N}\left(\mathbf{0}, \mathbf{R}_{q q}\right)$ is the additive Gaussian distributed quantization noise uncorrelated with $\overline{\mathbf{y}}$. The covariance matrix $\mathbf{R}_{q q}$ for the given channel is represented as $\mathbf{R}_{q q}=\mathbf{Q}_{a}\left(\mathbf{I}-\mathbf{Q}_{a}\right) \mathbb{E}\left[\overline{\mathbf{y}} \overline{\mathbf{y}}^{H}\right]$.

Digital Combiner (Active digital beamforming): Once the received signals are quantized, a linear digital combiner $\mathbf{v}_{k} \in$ $\mathbb{C}^{M_{R F} \times 1}$ is applied to $\mathbf{y}_{q}$ aiming at mitigating interference and quantization error. The retrieved symbol of user $k$ can be expressed as

$$
x_{k}=\mathbf{v}_{k}^{H} \mathbf{Q}_{a} \mathbf{W G} \mathbf{\Phi} \mathbf{H}_{r} \mathbf{P}^{\frac{1}{2}} \mathbf{S}+\mathbf{v}_{k}^{H} \mathbf{Q}_{a} \mathbf{W} \mathbf{z}+\mathbf{v}_{k}^{H} \mathbf{z}_{q} .
$$


We note that only imperfect CSI is available in the considered system due to the channel estimation errors. Define $\boldsymbol{\Theta}_{k}=\mathbf{G d i a g}\left(\mathbf{h}_{r, k}\right)$ as the cascaded CSI matrix from user $k$ to the BS via the RIS. Then the channel matrix $\boldsymbol{\Theta}_{k}$ in the presence of CSI errors is given by

$$
\boldsymbol{\Theta}_{k}=\hat{\boldsymbol{\Theta}}_{k}+\Delta \boldsymbol{\Theta}_{k},
$$

where $\hat{\boldsymbol{\Theta}}_{k}$ denotes the estimated cascaded CSI matrix ${ }^{1}$ and $\Delta \Theta_{k}$ represents the corresponding CSI error matrix. It is assumed that $\Delta \boldsymbol{\Theta}_{k}$ is statistically independent of $\hat{\boldsymbol{\Theta}}_{k}$ and follows the circularly symmetric complex Gaussian (CSCG) distribution $^{2}$, i.e., $\operatorname{vec}\left(\Delta \boldsymbol{\Theta}_{k}\right) \sim \mathcal{C N}\left(0, \sigma_{k}^{2} \mathbf{I}\right)$, where $\sigma_{k}^{2}$ is the variance of the individual CSI errors. Accordingly, we can rewrite (4) as follows

$$
x_{k}=\mathbf{v}_{k}^{H} \mathbf{Q}_{a} \mathbf{W}\left(\sum_{k}\left(\hat{\boldsymbol{\Theta}}_{k}+\Delta \boldsymbol{\Theta}_{k}\right) \boldsymbol{\varphi} \sqrt{p_{k}} s_{k}+\mathbf{z}\right)+\mathbf{v}_{k}^{H} \mathbf{z}_{q} .
$$

In this case, the mutual information conditioned on estimated channel matrix $\hat{\boldsymbol{\Theta}}_{k}$ can be written as $\mathcal{I}\left(s_{k} ; x_{k} \mid \hat{\boldsymbol{\Theta}}\right)$, where $\hat{\boldsymbol{\Theta}}=$ $\left\{\hat{\boldsymbol{\Theta}}_{k}\right\}_{k \in \mathcal{K}}$. According to [20], expanding $\mathcal{I}\left(s_{k} ; x_{k} \mid \hat{\boldsymbol{\Theta}}\right)$ in terms of the corresponding differential entropies results in

$$
\mathcal{I}\left(s_{k} ; x_{k} \mid \hat{\boldsymbol{\Theta}}\right)=\mathcal{H}\left(s_{k} \mid \hat{\boldsymbol{\Theta}}\right)-\mathcal{H}\left(s_{k} \mid x_{k}, \hat{\boldsymbol{\Theta}}\right) \text {. }
$$

Since $s_{k}$ follows the CSCG distribution with zero-mean and unit-variance, the first term on the right hand side (RHS) of (7) simplifies to $\log (2 \pi e)$ [21]. Regarding the second term on the RHS of (7), it is upper bounded by the entropy of a Gaussian random variable with variance equal to $\mathbb{E}\left[\left(s_{k}-g_{k} x_{k}\right)\left(s_{k}-g_{k} x_{k}\right)^{*} \mid \hat{\boldsymbol{\Theta}}\right]$ [22], where $g_{k} x_{k}$ denotes the (linear) MMSE estimation of $s_{k}$. Then, it yields the following inequality:

$$
\mathcal{H}\left(s_{k} \mid x_{k}, \hat{\boldsymbol{\Theta}}\right) \leq \log \left(2 \pi e\left(1-\frac{p_{k}\left|\mathbf{v}_{k}^{H} \mathbf{Q}_{a} \mathbf{W} \hat{\boldsymbol{\Theta}}_{k} \boldsymbol{\varphi}\right|^{2}}{\Gamma}\right)\right),
$$

where $\quad \Gamma \quad \triangleq \quad \sum_{j \neq k} p_{j}\left|\mathbf{v}_{k}^{H} \mathbf{Q}_{a} \mathbf{W} \hat{\boldsymbol{\Theta}}_{j} \boldsymbol{\varphi}\right|^{2}+$ $\sum_{j} p_{j} \sigma_{j}^{2} \boldsymbol{\varphi}^{H} \boldsymbol{\varphi}\left\|\mathbf{v}_{k}^{H} \mathbf{Q}_{a} \mathbf{W}\right\|^{2}+\sigma_{0}^{2}\left\|\mathbf{v}_{k}^{H} \mathbf{Q}_{a} \mathbf{W}\right\|^{2}+\mathbf{v}_{k}^{H} \mathbf{R}_{q q} \mathbf{v}_{k}$, and $\mathbf{R}_{q q}$ is the variance matrix of the additive quantized noise $\mathbf{z}_{q}$, expressed as

$$
\begin{aligned}
\mathbf{R}_{q q} \triangleq & \mathbf{Q}_{a}\left(\mathbf{I}-\mathbf{Q}_{a}\right) \operatorname{diag}\left(\sum_{j} p_{j} \mathbf{W} \boldsymbol{\Pi}_{j} \mathbf{W}^{H}\right. \\
& \left.+\sum_{j} p_{j} \sigma_{j}^{2} \boldsymbol{\varphi}^{H} \boldsymbol{\varphi} \mathbf{W} \mathbf{W}^{H}+\sigma_{0}^{2} \mathbf{W} \mathbf{W}^{H}\right),
\end{aligned}
$$

along with $\boldsymbol{\Pi}_{j} \triangleq \hat{\boldsymbol{\Theta}}_{j} \boldsymbol{\varphi} \varphi^{H} \hat{\boldsymbol{\Theta}}_{j}^{H}$. Hence, a lower bound of the conditional mutual information can be derived by inserting (8) into (7) which leads

$$
\begin{aligned}
\mathcal{I}\left(s_{k} ; x_{k} \mid \hat{\boldsymbol{\Theta}}\right) & \geq \mathcal{R}_{k}(\mathbf{W}, \mathbf{V}, \mathbf{p}, \mathbf{b}, \boldsymbol{\varphi}) \\
& \triangleq \log \left(1+\frac{p_{k}\left|\mathbf{v}_{k}^{H} \mathbf{Q}_{a} \mathbf{W} \hat{\boldsymbol{\Theta}}_{k} \boldsymbol{\varphi}\right|^{2}}{\Gamma}\right),
\end{aligned}
$$

\footnotetext{
${ }^{1}$ The linear minimum MSE (MMSE) method is adopted for estimating the cascaded CSI. Based on the linear MMSE estimator, the BS estimates the cascaded channel through a time division duplexing (TDD) operation including a two-phase pilot-based uplink training procedure by exploiting the correlation among the cascaded user-RIS-BS channels of different users [18] In fact, accurately estimating the cascaded channels is an important problem and still remains open in the RIS literature, which is beyond the scope of this compact Letter.

${ }^{2}$ Since the cascaded CSI can be estimated by applying the linear MMSE algorithm, in the presence of additive Gaussian noise, the resultant channel estimation errors obey the CSCG distribution [19].
}

where we define $\mathbf{V} \triangleq\left[\mathbf{v}_{1}, \cdots, \mathbf{v}_{K}\right]$ and $\mathbf{b} \triangleq\left[b_{1}, \cdots, b_{M_{R F}}\right]^{T}$ for convenience. Then, based on the sum of the lower bound of the mutual information over all users representing the sum of the achievable rate, we study the worst-case sum rate maximization optimization problem by performing the joint design of hybrid beamforming, bit allocation and transmit power control. Mathematically, the optimization problem is formulated as:

$$
\begin{aligned}
\max _{\mathbf{W}, \mathbf{V}, \mathbf{p}, \mathbf{b}, \boldsymbol{\varphi}} & \mathcal{R}=\sum_{k=1}^{K} \mathcal{R}_{k} \\
\text { s.t. } & b_{m} \in\left[b_{\min }, b_{\max }\right], b_{m} \in \mathbb{N}, \forall m, \\
& \|\mathbf{b}\|_{1} \leq M_{R F} b_{\text {avg }}, \\
& 0 \leq p_{k} \leq P_{k}, \forall k, \\
& \left|\varphi_{n}\right|=1, \forall n, \\
& |\mathbf{W}(m, i)|=1, \forall m, i,
\end{aligned}
$$

where $b_{\min }$ and $b_{\max }$ represent the minimum and maximum number of quantization bits of an $\mathrm{ADC}$, and $b_{\mathrm{avg}}$ stands for the average number of quantization bits for RADCs across the different RF chains. Constraint (11b) limits the range of the quantization bits for each RADC. The inequality constraint (11c) is added to provide the threshold of the total number of quantization bits for the system [3]. Constraint (11d) reflects the transmit power budget of user $k$, while (11e) and (11f) impose the constant-modulus constraints on all entries of the RIS passive beamforming vector and the active analog combining matrix, which are due to the practical implementations of the RIS elements and analog combiner, i.e., using low-cost phase-shifters.

\section{AlgORITHM DESCRIPTION}

Note that problem (11) is nonconvex and extremely challenging to tackle due to the coupled optimization variables in the objective function, bit allocation constraints over a discrete space and the constant-modulus constraints. To sum up, we face an NP-hard mixed-integer nonlinear programming (MINLP) problem. Aiming at solving problem (11), in this section, we first handle the logarithm term in the objective function based on the Lagrangian dual method. Thus, the objective function can be converted into an equivalent yet more tractable form. Then, we develop an efficient robust algorithm to jointly design beamformers and achieve resource allocation which is guaranteed to converge to a stationary solution of problem (11).

\section{A. Problem Transformation}

We note that the objective function of problem (11) involves complex logarithmic functions. To deal with it, we reformulate the objective function as

$$
\mathcal{R}_{1}=\sum_{k}\left(\log \left(1+\alpha_{k}\right)-\alpha_{k}+\frac{\tilde{\alpha}_{k}\left|\mathbf{v}_{k}^{H} \mathbf{Q}_{a} \mathbf{W} \hat{\boldsymbol{\Theta}}_{k} \boldsymbol{\varphi}\right|^{2}}{p_{k}\left|\mathbf{v}_{k}^{H} \mathbf{Q}_{a} \mathbf{W} \hat{\boldsymbol{\Theta}}_{k} \boldsymbol{\varphi}\right|^{2}+\Gamma}\right),
$$

where $\alpha_{k}$ refers to an auxiliary variable introduced for each ratio term in $\mathcal{R}_{k}$ and $\tilde{\alpha}_{k} \triangleq\left(1+\alpha_{k}\right) p_{k}$. Based on this, we have the following proposition.

Proposition 1: The original optimization problem (11) is equivalent to

$$
\max _{\mathbf{W}, \mathbf{V}, \mathbf{p}, \mathbf{b}, \boldsymbol{\varphi}, \boldsymbol{\alpha}} \mathcal{R}_{1} \quad \text { s.t. } \quad(11 b)-(11 f),
$$


where $\boldsymbol{\alpha}=\left[\alpha_{1}, \cdots, \alpha_{K}\right]^{T}$.

Proof: We note that the reformulated objective function $\mathcal{R}_{1}$ is concave in $\alpha$. Thus, by forcing the partial derivative of $\mathcal{R}_{1}$ with respect to $\alpha_{k}$ to zero, i.e., $\frac{\partial \mathcal{R}_{1}}{\partial \alpha_{k}}=0$, we obtain

$$
\alpha_{k}^{\star}=\frac{p_{k}\left|\mathbf{v}_{k}^{H} \mathbf{Q}_{a} \mathbf{W} \hat{\Theta}_{k} \varphi\right|^{2}}{\Gamma} .
$$

The objective function $\mathcal{R}$ can be obtained by substituting (14) back into the objective function $\mathcal{R}_{1}$.

Note that $\mathcal{R}_{1}$ is still in a multiple sum-of-ratio form. In light of the advanced fractional programming [23], by exploiting the quadratic transformation, we formulate Proposition 2 below.

Proposition 2: By introducing the auxiliary variable $\boldsymbol{\beta}=$ $\left[\beta_{1}, \cdots, \beta_{K}\right]^{T}$ with $\beta_{k} \in \mathbb{C}$ for each ratio in the last term of $\mathcal{R}_{1}$, the optimization problem (13) can be equivalently written as

$$
\max _{\mathbf{W}, \mathbf{V}, \mathbf{p}, \mathbf{b}, \boldsymbol{\varphi}, \boldsymbol{\alpha}, \boldsymbol{\beta}} \mathcal{R}_{2} \quad \text { s.t. } \quad(11 b)-(11 f),
$$

where the new objective function is

$$
\begin{aligned}
\mathcal{R}_{2}= & \sum_{k}\left(\log \left(1+\alpha_{k}\right)-\alpha_{k}+2 \sqrt{\tilde{\alpha}_{k}} \Re\left\{\beta_{k}^{*} \mathbf{v}_{k}^{H} \mathbf{Q}_{a} \mathbf{W} \hat{\boldsymbol{\Theta}}_{k} \boldsymbol{\varphi}\right\}\right. \\
& \left.-\left|\beta_{k}\right|^{2}\left(p_{k}\left|\mathbf{v}_{k}^{H} \mathbf{Q}_{a} \mathbf{W} \hat{\boldsymbol{\Theta}}_{k} \boldsymbol{\varphi}\right|^{2}+\Gamma\right)\right),
\end{aligned}
$$

and the optimal $\beta_{k}^{\star}$ satisfies

$$
\beta_{k}^{\star}=\frac{\sqrt{\tilde{\alpha}_{k}} \mathbf{v}_{k}^{H} \mathbf{Q}_{a} \mathbf{W} \hat{\boldsymbol{\Theta}}_{k} \boldsymbol{\varphi}}{p_{k}\left|\mathbf{v}_{k}^{H} \mathbf{Q}_{a} \mathbf{W} \hat{\boldsymbol{\Theta}}_{k} \boldsymbol{\varphi}\right|^{2}+\Gamma} .
$$

Proof: Detailed proof of this proposition is similar to that of Proposition 1, which is omitted due to page limitation.

Additionally, it is noteworthy that the bit allocation $b_{m}$ is represented by a discrete variable, which further complicates the problem. We subsequently relax the discrete integer constraint (11b) into a closed connected subset $\hat{b}_{m} \in$ $\left[b_{\min }, b_{\max }\right], \forall m$. For notational clarity, we define $\hat{\mathbf{b}}=$ $\left[\hat{b}_{1}, \cdots, \hat{b}_{M_{R F}}\right]^{T}$. Therefore, problem (15) is rewritten as :

$$
\begin{aligned}
\max _{\mathbf{W}, \mathbf{V}, \mathbf{p}, \hat{\mathbf{b}}, \boldsymbol{\varphi}, \boldsymbol{\alpha}, \boldsymbol{\beta}} & \mathcal{R}_{2} \\
\text { s.t. } & \hat{b}_{m} \in\left[b_{\min }, b_{\max }\right], \forall m, \\
& \|\hat{\mathbf{b}}\|_{1} \leq M_{R F} b_{\text {avg }}, \\
& (11 d)-(11 f) .
\end{aligned}
$$

Since the constraints in problem (18) are separable, we can solve problem (18) in an iterative manner with the aid of the alternating optimization. The key idea is to optimize one variable by fixing the others. Apparently, $\boldsymbol{\alpha}$ and $\boldsymbol{\beta}$ are first updated according to (14) and (17), respectively. In the next several subsections, we will focus on how to solve the other variables iteratively.

\section{B. Beamforming Design}

Optimizing RIS Passive Beamformer $\varphi$ : We focus on how to find a better beamforming vector $\varphi$ while holding the other variables fixed. The subproblem regarding to $\varphi$ is a unitmodulus constrained quadratic optimization problem, given by

$$
\begin{aligned}
\max _{\left|\varphi_{n}\right|=1} & \sum_{k} 2 \sqrt{\tilde{\alpha}_{k}} \Re\left\{\beta_{k}^{*} \mathbf{v}_{k}^{H} \mathbf{Q}_{a} \mathbf{W} \hat{\boldsymbol{\Theta}}_{k} \boldsymbol{\varphi}\right\} \\
& -\sum_{k}\left|\beta_{k}\right|^{2}\left(\sum_{j} p_{j}\left|\mathbf{v}_{k}^{H} \mathbf{Q}_{a} \mathbf{W} \hat{\boldsymbol{\Theta}}_{j} \boldsymbol{\varphi}\right|^{2}\right. \\
& \left.+\sum_{j} \sigma_{j}^{2} p_{j} \boldsymbol{\varphi}^{H} \boldsymbol{\varphi}\left\|\mathbf{v}_{k}^{H} \mathbf{Q}_{a} \mathbf{W}\right\|^{2}+\mathbf{v}_{k}^{H} \mathbf{F} \mathbf{v}_{k}\right),
\end{aligned}
$$

where $\mathbf{F} \triangleq \mathbf{Q}_{a}\left(\mathbf{I}-\mathbf{Q}_{a}\right) \operatorname{diag}\left(\sum_{j} p_{j} \mathbf{W} \boldsymbol{\Pi}_{j} \mathbf{W}^{H}+\right.$ $\left.\sum_{j} p_{j} \sigma_{j}^{2} \boldsymbol{\varphi}^{H} \boldsymbol{\varphi} \mathbf{W} \mathbf{W}^{H}\right)$. By appropriate rearrangement and defining some useful notations: $\hat{\boldsymbol{\Upsilon}}_{k} \triangleq \mathbf{Q}_{a} \mathbf{v}_{k} \mathbf{v}_{k}^{H} \mathbf{Q}_{a}$, $\check{\Upsilon}_{k} \triangleq \overline{\mathbf{V}}_{k} \overline{\mathbf{V}}_{k}^{H} \mathbf{Q}_{a}\left(\mathbf{I}-\mathbf{Q}_{a}\right)$ with $\overline{\mathbf{V}}_{k}=\operatorname{diag}\left(\mathbf{v}_{k}\right)$, problem (19) can be equivalently expressed as

$$
\max _{\left|\varphi_{n}\right|=1} \sum_{k} 2 \Re\left\{\operatorname{Tr}\left[\boldsymbol{\varphi}^{H} \mathbf{u}\right]\right\}-\sum_{k} \operatorname{Tr}\left[\boldsymbol{\varphi}^{H} \boldsymbol{\Lambda} \boldsymbol{\varphi}\right],
$$

where $\mathbf{u} \triangleq \sqrt{\tilde{\alpha}_{k}} \beta_{k} \hat{\boldsymbol{\Theta}}_{k}^{H} \mathbf{W}^{H} \mathbf{Q}_{a} \mathbf{v}_{k}, \quad \boldsymbol{\Lambda} \triangleq$ $\sum_{j}\left|\beta_{k}\right|^{2} p_{j}\left(\hat{\boldsymbol{\Theta}}_{j}^{H} \mathbf{W}^{H}\left(\hat{\boldsymbol{\Upsilon}}_{k}+\dot{\boldsymbol{\Upsilon}}_{k}\right) \mathbf{W} \hat{\boldsymbol{\Theta}}_{j}+\sigma_{j}^{2}\left\|\mathbf{v}_{k}^{H} \mathbf{Q}_{a} \mathbf{W}\right\|^{2} \mathbf{I}+\right.$ $\left.\sigma_{j}^{2} \mathbf{v}_{k}^{H} \mathbf{Q}_{a}\left(\mathbf{I}-\mathbf{Q}_{a}\right) \operatorname{diag}\left(\mathbf{W} \mathbf{W}^{H}\right) \mathbf{v}_{k} \mathbf{I}\right)$.

Under the separable unit-modulus constraints, we can readily solve this subproblem recursively by resorting to the oneiteration BCD-type algorithm presented in [24, Appendix B], where the one and only entry of $\varphi$ is updated with the others fixed at each step.

Optimizing Analog Combiner W: The corresponding subproblem for variable $\mathbf{W}$ is given by

$$
\begin{aligned}
\max _{\left|\mathbf{W}_{m, i}\right|=1} & \sum_{k}\left(2 \sqrt{\tilde{\alpha}_{k}} \Re\left\{\beta_{k}^{*} \mathbf{v}_{k}^{H} \mathbf{Q}_{a} \mathbf{W} \hat{\boldsymbol{\Theta}}_{k} \boldsymbol{\varphi}\right\}\right. \\
& \left.-\left|\beta_{k}\right|^{2}\left(p_{k}\left|\mathbf{v}_{k}^{H} \mathbf{Q}_{a} \mathbf{W} \hat{\boldsymbol{\Theta}}_{k} \boldsymbol{\varphi}\right|^{2}+\Gamma\right)\right) .
\end{aligned}
$$

For ease of addressing this problem, we rearrange it in a more tractable form as

$$
\max _{\left|\mathbf{W}_{m, i}\right|=1} \sum_{k}\left(2 \Re\left\{\operatorname{Tr}\left[\mathbf{W}^{H} \mathbf{\Upsilon}_{k}\right]\right\}-\operatorname{Tr}\left[\mathbf{W}^{H}\left(\hat{\mathbf{\Upsilon}}_{k}+\check{\mathbf{\Upsilon}}_{k}\right) \mathbf{W} \overline{\mathbf{\Upsilon}}_{k}\right]\right),
$$

where $\boldsymbol{\Upsilon}_{k} \triangleq \sqrt{\tilde{\alpha}_{k}} \beta_{k} \mathbf{Q}_{a} \mathbf{v}_{k} \boldsymbol{\varphi}^{H} \hat{\boldsymbol{\Theta}}_{k}^{H}$, and $\overline{\boldsymbol{\Upsilon}}_{k} \triangleq\left|\beta_{k}\right|^{2}\left(\sigma_{0}^{2} \mathbf{I}+\right.$ $\left.\sum_{j} p_{j}\left(\boldsymbol{\Pi}_{j}+\sigma_{j}^{2} \boldsymbol{\varphi}^{H} \boldsymbol{\varphi} \mathbf{I}\right)\right)$.

Similarly, we can utilize the same method as applied to (20) to tackle this problem.

Optimizing the Digital Combiner V: By keeping the other variables fixed, the subproblem with respect to $\mathbf{v}_{k}$ can be naturally decoupled among different $k$, and each one is an unconstrained quadratic optimization problem. There exists a closed-form solution of the optimal $\mathbf{v}_{k}^{\star}$ by examining the first order optimality condition. The solution can be written as

$$
\begin{aligned}
\mathbf{v}_{k}^{\star}= & \frac{\sqrt{\tilde{\alpha}_{k}} \beta_{k}^{*}}{\left|\beta_{k}\right|^{2}}\left(\sum_{j} p_{j}\left(\mathbf{Q}_{a} \mathbf{W} \boldsymbol{\Pi}_{j} \mathbf{W}^{H} \mathbf{Q}_{a}+\sigma_{j}^{2} \boldsymbol{\varphi}^{H} \boldsymbol{\varphi} \mathbf{Q}_{a} \mathbf{W} \mathbf{W}^{H} \mathbf{Q}_{a}\right)\right. \\
& \left.+\sigma_{0}^{2} \mathbf{Q}_{a} \mathbf{W} \mathbf{W}^{H} \mathbf{Q}_{a}+\mathbf{R}_{q q}\right)^{-1} \mathbf{Q}_{a} \mathbf{W} \hat{\Theta}_{k} \boldsymbol{\varphi}
\end{aligned}
$$

\section{Bit Allocation}

Here, we continue to solve the subproblem with regard to $\hat{b}$ with the other variables fixed. It should be pointed out that in nature the corresponding objective function $\mathcal{R}_{2}$ is nonconcave in $\hat{\mathbf{b}}$. To handle this issue, we employ the successive concave approximation (SCA) method for the objective function $\mathcal{R}_{2}$. Then, in the $t$-th iteration of the SCA, we construct the following concave surrogate function:

$$
f^{t}(\hat{\mathbf{b}})=\mathcal{R}_{2}\left(\hat{\mathbf{b}}^{t}\right)+\nabla_{\hat{\mathbf{b}}}^{T} \mathcal{R}_{2}\left(\hat{\mathbf{b}}^{t}\right)\left(\hat{\mathbf{b}}-\hat{\mathbf{b}}^{t}\right)-\xi\left\|\hat{\mathbf{b}}-\hat{\mathbf{b}}^{t}\right\|^{2},
$$

where $\nabla_{\hat{\mathbf{b}}} \mathcal{R}_{2}\left(\hat{\mathbf{b}}^{t}\right)$ is the partial derivative of $\mathcal{R}_{2}(\hat{\mathbf{b}})$ with respect to $\hat{\mathbf{b}}$ at the current point $\hat{\mathbf{b}}^{t}, \xi>0$ can be any constant, and the term $\xi\left\|\hat{\mathbf{b}}-\hat{\mathbf{b}}^{t}\right\|^{2}$ is used to ensure strong concavity of the surrogate function $f^{t}(\hat{\mathbf{b}})$. Therefore, the resulting linearly constrained quadratic optimization problem that needs to be solved in the $t$-th iteration is given by

$$
\hat{\mathbf{b}}^{t+1}=\arg \max _{\hat{\mathbf{b}}} f^{t}(\hat{\mathbf{b}}) \quad \text { s.t. }(18 b),(18 c),
$$

which can be optimally solved via off-the-shelf solvers such as CVX [25]. To obtain an integer solution for the optimal bit allocation in each ADC, we round $\hat{b}_{n}$ to its nearby integer based on the method in [3]. 


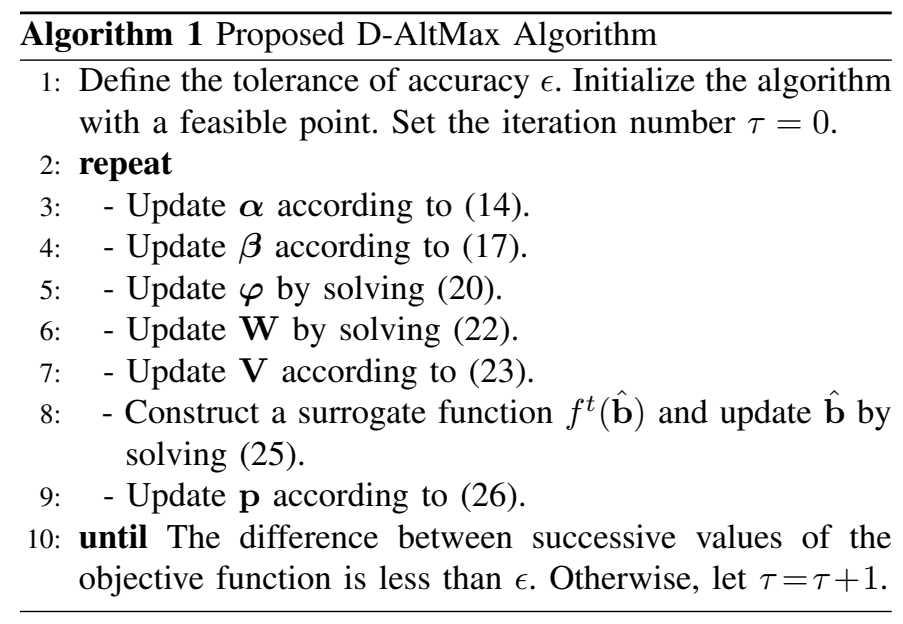

\section{Power Control}

Since the power control subproblem reduces to a concave optimization problem along with linear constraints, it can be uniquely solved by checking the first-order optimality condition. As such, the optimal $p_{k}^{\star}$ is given by

$$
p_{k}^{\star}=\min \left\{P_{k},\left(\Re\left\{\sqrt{1+\alpha_{k}} \beta_{k}^{*} \mathbf{v}_{k}^{H} \mathbf{Q}_{a} \mathbf{W} \hat{\boldsymbol{\Theta}}_{k} \boldsymbol{\varphi}\right\} / \omega_{k}\right)^{2}\right\},
$$

where $\omega_{k} \triangleq \sum_{j}\left|\beta_{j}\right|^{2}\left(\operatorname{Tr}\left[\mathbf{W} \Pi_{k} \mathbf{W}^{H} \check{\Upsilon}_{j}+\sigma_{k}^{2} \boldsymbol{\varphi}^{H} \boldsymbol{\varphi} \mathbf{W} \mathbf{W}^{H} \check{\mathbf{\Upsilon}}_{j}\right]+\right.$ $\left.\left|\mathbf{v}_{j}^{H} \mathbf{Q}_{a} \mathbf{W} \hat{\boldsymbol{\Theta}}_{k} \boldsymbol{\varphi}\right|^{2}+\sigma_{k}^{2} \boldsymbol{\varphi}^{H} \boldsymbol{\varphi}\left\|\mathbf{v}_{j}^{H} \mathbf{Q}_{a} \mathbf{W}\right\|^{2}\right)$.

Finally, the proposed decoupled alternating maximization (D-AltMax) algorithm is summarized in Algorithm 1.

\section{E. Convergence and Complexity}

It is noteworthy that each subproblem of the D-AltMax algorithm is nondecreasing and guaranteed to converge to its stationary point. Therefore, the objective value of (18) is ensured to be nondecreasing at each iteration. As a result, the D-AltMax algorithm will converge towards a stationary solution to problem (18). Since problem (18) and problem (11) are equivalent, the obtained solution is also a stationary point of the original problem (11).

We then analyze the computational complexity of the DAltMax algorithm, which is measured by the number of complex multiplication under the assumption $M_{r} \gg M_{R F} \geq$ $K$.Considering only the dominant components of the calculations, the complexity is dominated by the update of $\mathbf{W}$, $\mathbf{V}, \varphi$ and $\mathbf{b}$. The corresponding computational costs of $\mathbf{W}$ and $\varphi$ are $\mathcal{O}\left(M_{r}^{2} M_{R F}^{2}\right)$ and $\mathcal{O}\left(N^{2}\right)$, respectively [24]. The calculation of $\mathbf{V}$ involves the inverse of the matrix at a complexity of $\mathcal{O}\left(K M_{R F}^{3}\right)$. The computation complexity of updating $\mathbf{b}$ is dominated by computing the Jacobian matrix and using the toolbox CVX to solve problem (25), which leads to $\mathcal{O}\left(K M_{r} M_{R F} N+M_{R F}{ }^{3.5} \log (1 / \epsilon)\right)$ to get an $\epsilon$-optimal solution [26]. Therefore, the overall complexity of the proposed algorithm in each iteration can be expressed as $\mathcal{O}\left(M_{r}^{2} M_{R F}^{2}+\right.$ $\left.N^{2}+K M_{R F}^{3}+T\left(K M_{r} M_{R F} N+M_{R F}{ }^{3.5} \log (1 / \epsilon)\right)\right)$, where we let $T$ denote the number of iterations of the SCA method.

\section{Simulation Results}

In this section, we evaluate the performance of our proposed design via Monte-Carlo simulation. The BS and RIS are located at $(5 \mathrm{~m}, 0,0)$ and $(0,20 \mathrm{~m}, 30 \mathrm{~m})$, respectively. There are $K=6$ users uniformly and randomly distributed in a circle centered at $(10 \mathrm{~m}, 25 \mathrm{~m}, 0)$ with radius $5 \mathrm{~m}$. In addition, we assume a Rician fading channel model comprising a line-ofsight (LOS) path and a number of non-LOS (NLOS) paths
[12]. Specifically, the user-RIS channel and RIS-BS channel are modeled as follows

$$
\begin{aligned}
& \mathbf{h}_{r, k}=\sqrt{\frac{N}{L_{I}+1}}\left(\varrho_{k 0} \mathbf{a}_{r}\left(\psi_{k 0}^{a}, \psi_{k 0}^{e}\right)+\sum_{l=1}^{L_{I}} \varrho_{k l} \mathbf{a}_{r}\left(\psi_{k l}^{a}, \psi_{k l}^{e}\right)\right), \\
& \mathbf{G}=\sqrt{\frac{M_{r} N}{L_{B}+1}}\left(\eta_{0} \mathbf{a}_{r}\left(\phi_{0}\right) \mathbf{a}_{t}^{H}\left(\vartheta_{0}^{a}, \vartheta_{0}^{e}\right)+\sum_{l=1}^{L_{B}} \eta_{l} \mathbf{a}_{r}\left(\phi_{l}\right) \mathbf{a}_{t}^{H}\left(\vartheta_{l}^{a}, \vartheta_{l}^{e}\right)\right),
\end{aligned}
$$

where $L_{I}$ and $L_{B}$ denote the NLOS propagation paths for the user-RIS link and RIS-BS link, respectively. $\varrho_{k 0}\left(\eta_{0}\right) \sim$ $\mathcal{C N}\left(0,10^{-0.1 \kappa}\right)$ and $\varrho_{k l}\left(\eta_{l}\right) \sim \mathcal{C N}\left(0,10^{-0.1 \varpi}\right)$ denote the complex gain associated with the LOS link and the $l$-th NLOS link, respectively, where $\kappa$ and $\varpi$ express the path-loss over a distance $d$ between the transmitter and the receiver, satisfying $\kappa=61.4+20 \log (d)+\varepsilon_{1}$ with $\varepsilon_{1} \sim \mathcal{C N}\left(0, \sigma_{1}^{2}\right)$ and $\varpi=72+29.2 \log (d)+\varepsilon_{2}$ with $\varepsilon_{2} \sim \mathcal{C N}\left(0, \sigma_{2}^{2}\right)$. We set $\sigma_{1}=5.8 \mathrm{~dB}$ and $\sigma_{2}=8.7 \mathrm{~dB}$ [27]. Furthermore, $\psi_{k l}^{a}\left(\psi_{k l}^{e}\right)$ and $\vartheta_{l}^{a}\left(\vartheta_{l}^{e}\right)$ are the azimuth (elevation) angle-of-arrival (AoA) and azimuth (elevation) angle-of-departure (AoD) associated with the RIS, respectively, while $\phi_{l}$ represents the AoA associated with the BS. Also, $\mathbf{a}_{r}$ and $\mathbf{a}_{t}$ denote the array response vectors associated with the receiver and transmitter, respectively. Unless stated otherwise, we assume $M_{r}=64$, $M_{R F}=6, L_{B}=L_{I}=7, N=100, b_{\min }=1, b_{\max }=8$, $b_{\text {avg }}=3, \sigma_{0}^{2}=-90 \mathrm{dBm}$ and $P_{k}=P_{\max }=30 \mathrm{dBm}, \forall k$. According to the statistical cascaded CSI error model [28], $\sigma_{k}^{2}$ is defined as $\sigma_{k}^{2}=\mu_{k}^{2}\left\|\operatorname{vec}\left(\hat{\boldsymbol{\Theta}}_{k}\right)\right\|^{2}$, where $\mu_{k}^{2} \in[0,1)$ is the normalized CSI error measuring the CSI uncertainty level. For convenience, we assume that the normalized CSI errors of different users are identical, i.e., $\mu_{k}^{2}=\mu^{2}$.

We contrast the performance of the proposed D-AltMax algorithm to the following benchmark schemes: 1) RandomRIS, where the reflection coefficients of the RIS are randomly selected; 2) Nonrobust, where the effect of channel estimation error is not taken into consideration under imperfect CSI; 3) MRC-case, where the maximum ratio combining (MRC) based digital combiner is adopted [2]; 4) Full-power, where the users transmit signals with full power; 5) Fixed-bit ADCs, where the LADCs with uniform quantization bits are implemented at the BS; 6) ZF-case, where the zero forcing (ZF) digital combiner is used at the BS [2].

In Fig. 2, we illustrate the convergence performance. It can be observed that the proposed D-AltMax algorithm attains the maximum objective function value within a few iterations.

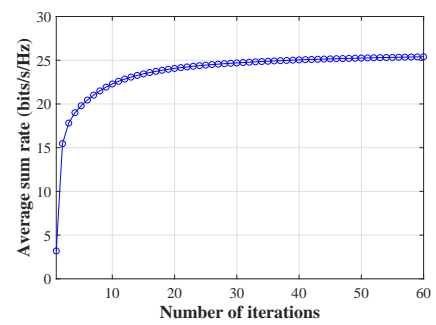

Fig. 2. Convergence of Algorithm 1.

Fig. 3(a) shows the average sum rate versus the maximum transmit power $P_{\max }$ over 100 random channel realizations. It can be seen that the proposed D-AltMax algorithm considerably outperforms all the benchmarks, and the performance gains become more significant as $P_{\max }$ increases. The result confirms the benefit of the joint design of bit allocation, power control and beamforming in both interference suppression and signal enhancement, especially when the transmit power (interference) increases. Fig. 3(b) depicts the average sum rate versus the normalized CSI error variance $\mu^{2}$. We observe 


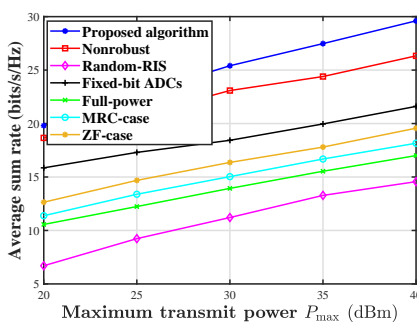

(a)

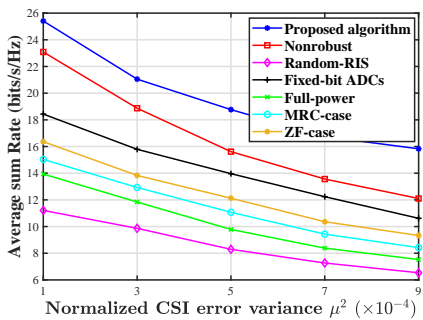

(b)
Fig. 3. Average sum rate versus (a) maximum transmit power $P_{\max }$, (b) normalized CSI error variance $\mu^{2}$.

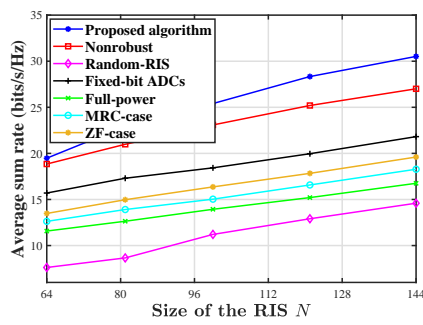

(a)

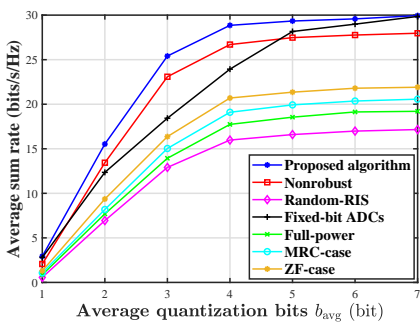

(b)
Fig. 4. Average sum rate versus (a) the size of RIS elements $N$, (b) the number of average quantization bits $b_{\text {avg. }}$.

that the average sum rate decreases with the degradation of CSI accuracy in all schemes. Particularly, compared to the proposed scheme, the nonrobust scheme results in notable performance degradation with the increase of $\mu^{2}$ due to the fact that the nonrobust scheme is more sensitive to the CSI errors. This result corroborates the robustness of our proposed algorithm and illustrates the necessity of robust designs in RISaided communication systems by considering the CSI errors.

In Fig. 4(a), the average sum rate of the system is plotted for different RIS element values. We note that the average sum rate increases monotonically upon increasing $N$ in all cases. Furthermore, it is readily seen that the performance gap becomes more pronounced upon increasing the number of RIS elements. In particular, in Fig. 4(b) we plot the average sum rate versus the average number of quantization bits $b_{\text {avg }}$, where increasing the average number of quantization bits $b_{\text {avg }}$ offers a prominent improvement of the average sum rate. Observe that the proposed D-AltMax algorithm provides the highest average sum rate in all quantization regimes. We note that the performance gap between the proposed DAltMax algorithm and the Fix-bit ADCs case increases in the moderate-resolution regime (e.g., $b_{\text {avg }}=3$ or 4 ). The reason is that the proposed flexible RADC architecture has sufficient freedom to adaptively allocate quantization bits relying on the specific propagation conditions.

\section{CONCLUSION}

In conclusion, we incorporated an RIS in the uplink of multiuser MIMO systems relying on realistic imperfect CSI. We then maximized the worst-case sum rate by jointly optimizing the transmit power, robust beamforming, and the number of $\mathrm{ADC}$ quantization bits under practical constraints. We employed the Lagrangian dual method and fractional programming techniques to transform the optimization problem formulated into an equivalent but more tractable form and then conceived a new D-AltMax iterative algorithm for solving it. Our simulation results confirmed the supremacy of the proposed scheme over the benchmark schemes. As future work, it is worth investigating the application of RISs as refractors. We would consider the extension of the joint design to a system where obstacles are empowered by the RIS architecture and assist data transmission via smart refraction.

\section{REFERENCES}

[1] J. Mo et al., "Hybrid architectures with few-bit ADC receivers: Achievable rates and energy-rate tradeoffs," IEEE Trans. Wireless Commun., vol. 16, no. 4, pp. 2274-2287, April 2017.

[2] Y. Wang et al., "Stochastic hybrid combining design for quantized massive MIMO systems," IEEE Trans. Veh. Technol., vol. 69, no. 12, pp. 16224-16229, Dec. 2020

[3] X. Chen et al., "Joint user scheduling and resource allocation for millimeter wave systems relying on adaptive-resolution ADCs," IEEE Trans. Veh. Technol., doi: 10.1109/TVT.2021.3112930.

[4] W. B. Abbas et al., "Millimeter wave receiver efficiency: A comprehensive comparison of beamforming schemes with LADCs," IEEE Trans. Wireless Commun., vol. 16, no. 12, pp. 8131-8146, Dec. 2017.

[5] M. Di Renzo et al., "Smart radio environments empowered by reconfigurable AI meta-surfaces: An idea whose time has come," EURASIP J. Wireless Commun. Netw., vol. 129, no. 1, pp. 1-20, 2019.

[6] D. Xu et al., "Resource allocation for IRS-assisted full-duplex cognitive radio systems," IEEE Trans. Commun., vol. 68, no. 12, pp. 7376-7394, Dec. 2020.

[7] S. Li et al., "Reconfigurable intelligent surface assisted UAV communication: Joint trajectory design and passive beamforming," IEEE Wireless Commun. Lett., vol. 9, no. 5, pp. 716-720, May 2020.

[8] L. Yang et al., "On the performance of RIS-assisted dual-hop UAV communication systems," IEEE Trans. Veh. Technol., vol. 69, no. 9, pp. 10385-10390, Sep. 2020

[9] M. Nemati et al.,"Modeling RIS empowered outdoor-to-indoor communication in mmWave cellular networks," IEEE Trans. Commun., doi: 10.1109/TCOMM.2021.3104878

[10] C. Pradhan et al., "Hybrid precoding design for reconfigurable intelligent surface aided mmWave communication systems," IEEE Wireles Commun. Lett., vol. 9, no. 7, pp. 1041-1045, Jul. 2020.

[11] P. Wang et al., "Intelligent reflecting surface-assisted millimeter wave communications: Joint active and passive precoding design," IEEE Trans. Veh. Technol., vol. 69, no. 12, pp. 14960-14973, Dec. 2020.

[12] P. Wang et al., "Joint transceiver and large intelligent surface design for massive MIMO mmWave systems," IEEE Trans. Wireless Commun., vol. 20, no. 2, pp. 1052-1064, Feb. 2021.

[13] Q. Wu et al., "Intelligent reflecting surface aided wireless communications: A tutorial," IEEE Trans. Commun., vol. 69, no. 5, pp. 3313-3351, May 2021.

[14] C. Pradhanet et al. "Hybrid precoding design for reconfigurable intelligent surface aided mmWave communication systems," IEEE Wireless Commun. Lett., vol. 9, no. 7, pp. 1041-1045, Jul. 2020

[15] Z. Xu et al., "Analog combining in intelligent reflecting surface assisted system with low-resolution ADCs," Journal of Physics: Conference Series, vol.1864, Nov. 2020.

[16] K. Zhi et al., "Uplink achievable rate of intelligent reflecting surfaceaided millimeter-wave communications with low-resolution ADC and phase noise," IEEE Wireless Commun. Lett., vol. 10, no. 3, pp. 654658, Mar. 2021

[17] Y. Xiu et al., "Uplink achievable rate maximization for reconfigurable intelligent surface aided millimeter wave systems with resolution-adaptive ADCs," IEEE Wireless Commun. Lett, vol. 10, no. 8, pp. 1608-1612, Aug. 2021.

[18] Z. Wang et al., "Channel estimation for intelligent reflecting surface assisted multiuser communications: Framework, algorithms, and analysis," IEEE Trans. Wireless Commun., vol. 19, no. 10, pp. 6607-6620, Oct. 2020

[19] Z. Zhou et al., "Joint transmit precoding and reconfigurable intelligent surface phase adjustment: A decomposition-aided channel estimation approach," IEEE Trans. Commun., vol. 69, no. 2, pp. 1228-1243, Feb. 2021.

[20] M. Medard, "The effect upon channel capacity in wireless communications of perfect and imperfect knowledge of the channel," IEEE Trans. Inf. Theory, vol. 46, no. 3, pp. 933-946, May 2000.

[21] T. M. Cover et al., Elements of Information Theory: Wiley Series in Telecommunications. New York, NY, USA: Wiley, 1991.

[22] Y. Cai et al., "Robust joint hybrid transceiver design for millimeter wave full-duplex MIMO relay systems," IEEE Trans. Wireless Commun., vol. 18, no. 2, pp. 1199-1215, Feb. 2019.

[23] K. Shen et al., "Fractional programming for communication systemsPart I: Power control and beamforming," IEEE Trans. Signal Process., vol. 66, no. 10, pp. 2616-2630, May 2018.

[24] Q. Shi et al., "Spectral efficiency optimization for mmWave multiuser MIMO systems," IEEE J. Sel. Toptics Signal Process., vol. 12, no. 3, pp. 455-468, Jun. 2018

[25] M. Grant et al., "CVX: Matlab software for disciplined convex programming, version 2.1," http://cvxr.com/cvx, Mar. 2014.

[26] Y. Ye, Interior Point Algorithms: Theory and Analysis. New York: Wiley, 1997.

[27] M. R. Akdeniz et al., "Millimeter wave channel modeling and cellular capacity evaluation,“ IEEE J. Sel. Areas Commun.,vol. 32, no. 6, pp. 1164-1179, Jun. 2014.

[28] S. Hong et al., "Robust transmission design for intelligent reflecting surface-aided secure communication systems with imperfect cascaded CSI," IEEE Trans. Wireless Commun., vol. 20, no. 4, pp. 2487-2501, Apr. 2021. 